\title{
Analysis of neurology consultations in hospitalized patients with COVID-19
}

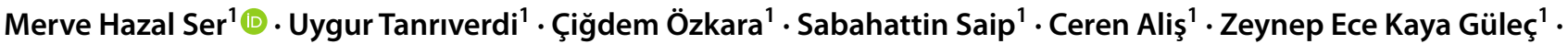 \\ Gözde Nezir ${ }^{1}$ - Esra Koçhan Kızılkılıç ${ }^{1}$. Merve Aktan Süzgün ${ }^{1} \cdot$ Hikmet Abbaszade ${ }^{1}$ · Şermin Börekçi ${ }^{2}$. \\ Rıdvan Karaali ${ }^{3}$ - Osman Kızılkılıçç $\cdot$ Ayşegül Gündüz ${ }^{1} \cdot$ Feray Karaali Savrun ${ }^{1}$
}

Received: 17 July 2021 / Accepted: 29 October 2021 / Published online: 10 January 2022

(c) Belgian Neurological Society 2021

\begin{abstract}
Objective The aim of this study was to evaluate patients who were hospitalized with a diagnosis of COVID-19 and were consulted by neurology during their hospital stay.

Methods All files of patients with COVID-19 who were admitted to Cerrahpasa Medical Faculty Hospital between March 11th and December 31st, 2020 were retrospectively reviewed, and files of patients who consulted by neurology during their stay were included. Demographic and clinical characteristics, neurologic diagnosis, outcome and related laboratory data were extracted from electronic medical records and analyzed. Patients were categorized into the first wave and second wave according to the date of hospitalization.

Results A total of 2257 patients were hospitalized for COVID-19; among them, 127 were consulted by a neurologist during their hospital stay. Fifteen patients received a consultation for possible drug interactions. Among the remaining 112 patients, the reason for neurology consultation was i. exacerbation of a neurological comorbidity vs ii. new-onset neurological manifestations. The median age was $68.5 \pm 14.2$ years, and $60.7 \%$ were men. Dementia and stroke were the leading neurological comorbidities. COVID-19 disease was more severe in the patients with the new-onset neurological comorbidity than in patients with exacerbation of a neurological comorbidity $(p=0.07)$. Serum creatinine kinase levels were higher in the newonset patient group $(p<0.05)$. Exacerbation of previous neurological disease or new neurological impairment were jointly and severely related to high mortality (overall $35 / 112$ vs $275 / 2145, p<0.001$; exacerbation $12 / 45$ vs $275 / 2145 p<0.01$; new-onset $23 / 67$ vs $275 / 2145, p<0.001$ ).
\end{abstract}

Conclusion Serious neurological involvement is relatively uncommon in hospitalized patients with COVID-19 and is associated with increased mortality.

Keywords COVID-19 $\cdot$ Neurology $\cdot$ Consultation $\cdot$ Inpatient $\cdot$ Mortality

\section{Introduction}

Merve Hazal Ser

hazalyilmaz@istanbul.edu.tr; mhazalyilmaz90@gmail.com

Uygur Tanriverdi

uygur.tanriverdi@istanbul.edu.tr

1 Department of Neurology, Cerrahpaşa Medical Faculty, Istanbul University-Cerrahpaşa, Istanbul, Turkey

2 Department of Pulmonary Disease, Cerrahpaşa Medical Faculty, Istanbul University-Cerrahpaşa, Istanbul, Turkey

3 Department of Infectious Disease, Cerrahpaşa Medical Faculty, Istanbul University-Cerrahpaşa, Istanbul, Turkey

4 Department of Radiology, Cerrahpaşa Medical Faculty, Istanbul University-Cerrahpaşa, Istanbul, Turkey
Since the discovery of a novel coronavirus, severe acute respiratory syndrome coronavirus 2 (SARS-CoV-2), in December 2019, its spread worldwide has caused serious consequences affecting billions of people on earth [1]. The World Health Organization (WHO) declared the novel coronavirus (COVID-19) outbreak a global pandemic on March 11th, 2020 [2]. The total number of patients with COVID-19 in Turkey is $2,55 ., 633$, and the total number of deaths is 28,213 as of February 24, 2021 [3].

SARS-CoV-2 is a single-stranded RNA virus in the Betacoronavirus species class known for its potential to cause neurological involvement. [4] SARS-COV-2 binding protein 
can cross the blood-brain barrier in mice after intranasal and intravenous administration [5]. It is not yet clear whether SARS-CoV-2 is a neurotropic virus for humans, although it shares close homology to SARS-CoV-1, which is reliably detected in human neural specimens [6]. Several routes are offered for viral neuroinvasion, including transsynaptic transfer across infected neurons, entry via the olfactory nerve, infection of vascular endothelium or leukocyte migration across the blood-brain barrier [7].

Reports of neurological manifestations of SARS-CoV-2 are increasing. Headache and dizziness are the most common neurologic manifestations of COVID-19; indeed, they are historically associated with viral infections $[8,9]$. More serious involvement, such as encephalopathy, meningoencephalitis, stroke, peripheral nerve disorders, and myopathy, has also been reported [10-13]. Patients with a previous diagnosis of chronic neurological diseases are supposedly at increased risk of serious COVID-19 infection [14]. For example, dementia is a risk factor for mortality independent of age [15]. However, Parkinson's disease-related morbidity and mortality did not differ from the general population in mild to moderate disease severity, whereas higher mortality rates were associated with longer disease duration [16, 17]. Although the risk of COVID-19 in immune-related neurological disorders is controversial, there is evidence suggesting that it depends upon disease severity and the type of immunotherapy. [18, 19] For these reasons, we aimed to review the following from the files of patients who were hospitalized with COVID-19 between March 11, 2020, and December 31, 2020: (i) neurological conditions that developed during the course of COVID-19 and (ii) neurological disorders that were present before the development of COVID-19. Our first goal was to analyze the risk factors for the development of neurological conditions, whereas the secondary goal was to determine the contribution of comorbid neurological disorders to prognosis.

\section{Patients and methods}

\section{Study design}

This is a retrospective, cross-sectional study at Istanbul University-Cerrahpasa, Cerrahpasa Medical Faculty. We reviewed all the medical records of the patients who were hospitalized due to COVID-19 infection and consulted with neurologists who had more than 3 years of experience from March 11, 2020, to December 31, 2020. The diagnosis of COVID-19 was made by the detection of SARS-CoV-2 RNA with real-time reverse transcription-polymerase chain reaction (rt-PCR) of throat swab samples and/or probable pneumonia findings in thorax computed tomography (CT).
We retrieved the data regarding age, sex, previous neurological disorders, present neurological complaints and neurological examination findings from the medical files.

The time interval between hospitalization and neurology consultation and the severity of the disease were noted for each patient. The severity of COVID-19 was defined according to the World Health Organization COVID-19 guide, which divides the severity of disease into four categories at the neurology consultation date: mild (only symptoms related to COVID-19), moderate (including signs of pneumonia), severe (severe pneumonia with decreased saturation levels) and critical (acute respiratory distress syndrome) [20]. This classification was modified into two categories: mild and moderate vs. severe and critical.

We noted the findings of radiological investigations, including thorax computerized tomography (CT), brain $\mathrm{CT}$, brain magnetic resonance imaging (MRI) and MR angiography (MRA). Electroencephalography (EEG) and cerebrospinal fluid analysis (CSF) were also noted if they were performed.

Reasons for consultation were grouped as altered mental status, stroke, extrapyramidal symptoms, headache, seizure, vertigo/dizziness, sensory disturbances, cranial neuropathy, retinitis, myasthenia gravis (MG), hiccups, syncope, restless leg syndrome, neuralgia and motor neuron disease. Consultations seeking recommendations about drug interactions without any new neurological complaints or worsening of an ongoing neurological disorder were also noted.

The primary outcome of the analysis was designated as follows: (1) deterioration of an ongoing neurological disorder related to COVID-19 and (2) new neurological disorder caused by COVID-19. The outcome of COVID19 disease itself was also noted as exitus or discharge to home.

Key laboratory investigations, including ferritin, D-dimer, fibrinogen, thrombocytes and creatinine kinase, were documented.

The treatment protocol for COVID-19 was noted, which included monotherapy or different combinations of the following medications in Turkey: hydroxychloroquine, azithromycin, favipiravir, lopinavir/ritonavir, oseltamivir, tocilizumab, corticosteroids, anticoagulants, and remdesivir.

Patients were categorized into the first wave (March 11-August 31,2020) and the second wave (September 1-December 31, 2020) according to the date of hospitalization because the indications of hospitalization were different between these dates in our hospital. In the first wave, all patients with a confirmed COVID-19 infection were hospitalized, in contrast to the second wave, in which only patients with stage 3 or 4 diseases or with stage 1 or 2 disease plus older age and comorbidities were hospitalized. 


\section{Statistical analysis}

Continuous variables are indicated as the median and interquartile range (IQR). Categorical variables were indicated as $n(\%)$. Shapiro-Wilk test was performed for normality. As all the data were nonparametric, we used the $\chi^{2}$ test for categorical variables and the Mann-Whitney $U$ test for continuous variables to compare between groups. Statistical significance was set at a $P$-value $\leq 0.05$. All calculations were made using IBM SPSS software version 20 (International Business Machines Corp., New Orchard Road, Armonk, NY, USA).

\section{Ethics approval}

This study was approved by the Ethics Committee of Istanbul University-Cerrahpasa, Cerrahpasa Medical Faculty and the National Ministry of Health.

\section{Results}

A total of 2257 patients were hospitalized due to COVID19 infection between March 11, 2020, and December 31, 2020 , in our hospital. The median age was 58 (46-70) years, and $55.1 \%$ of the patients were men. Of these patients, 1340 were hospitalized during the first wave, and 917 were hospitalized during the second wave. Among them, 132 (9.9\%) died in the first wave during the hospital stay, whereas $178(19.4 \%)$ died during the second wave $(p<0.001)$.

Of 2257 patients, 127 were consulted by a neurologist during their hospital stay. Fifteen (11.8\%) patients were consulted by neurology for probable drug interactions. They had neither a deterioration nor a new symptom of their neurological status. Therefore, these patients were excluded from further analysis (Fig. 1).

Among the remaining 112 patients, the median age was 68.5 (57.25-76.75) years, and $60.7 \%$ of these patients were men. Forty-six patients $(41.1 \%)$ received consultation during the first wave, and 66 patients $(58.9 \%)$ received consultation during the second wave. Fifteen patients (32.6\%) died in the first wave, whereas 20 patients $(30.3 \%)$ died in the second wave. The mortality rate was higher for patients who had a new neurological symptom or had an exacerbation in both the first and second waves and in total (first wave $15 / 46$ vs $132 / 1294, p<0.001$; second wave $158 / 851$ vs $20 / 66, p=0.02$; overall $35 / 112$ vs $275 / 2145, p<0.001$ ).

The clinical and demographic data of the 112 patients are shown in Table 1. Hypertension was the most common

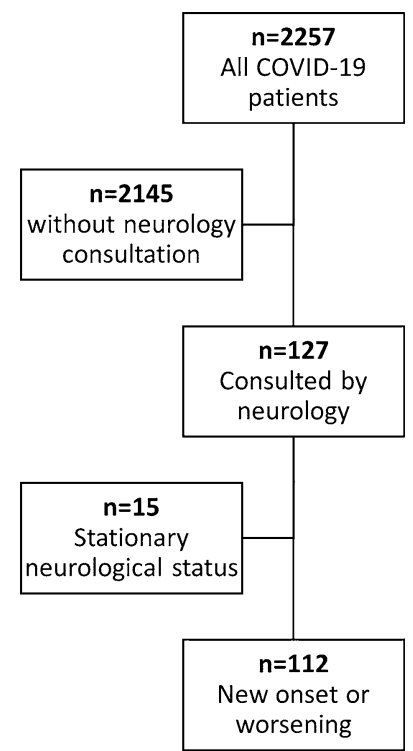

Fig. 1 Flow chart of patients included in the study

Table 1 Demographics and risk factors

\begin{tabular}{ll}
\hline & All patients $(n=112)$ \\
\hline Age (years) median (IQR) & $68.5(57.25-76.75)$ \\
Gender (male) n (\%) & $68(60.7)$ \\
Consultation day after hospitalization (days) & $3(1-7)$ \\
$\quad$ median (IQR) & \\
Hypertension $n(\%)$ & $36(32.1)$ \\
Diabetes mellitus $n(\%)$ & $21(18.8)$ \\
Malignancy $n(\%)$ & $17(15,2)$ \\
Cardiac disorders $n(\%)$ & $23(20,5)$ \\
Pulmonary disorders $n(\%)$ & $11(9.8)$ \\
Chronic renal failure $n(\%)$ & $11(9.8)$ \\
\hline
\end{tabular}

comorbid systemic disease. The median duration of hospitalization before the consultation was $3(1-7)$ days.

Fifty-seven patients had neurological comorbidities. Twenty-two died during their hospital stay. Dementia and stroke were the leading neurological disorders. The relationship between neurological comorbidity subtypes and death was examined for each, and no statistical significance was found. Table 2 shows previous neurological diagnoses of consulted patients.

Patients were separated into groups of (i) exacerbation of a neurological comorbidity and (ii) new-onset neurological manifestation associated with COVID-19 infection. There were no differences between risk factors and exitus between the two patient groups although COVID-19 severity was higher in the new-onset group $(p=0,07)$ (Table 3 ). 
Table 2 Neurological comorbidities

\begin{tabular}{lll}
\hline & $n(\%)^{*}$ & Exitus $n(\%)^{* *}$ \\
\hline Neurological comorbidities $n(\%)$ & $57(50,9)$ & $22(38,6)$ \\
Stroke & $16(14,3)$ & $5(31,3)$ \\
Dementia & $18(16,1)$ & $7(38,9)$ \\
Intracranial mass lesion & $9(8)$ & $5(55,6)$ \\
Parkinsonism & $10(8,9)$ & $3(30)$ \\
Myasthenia gravis & $4(3,6)$ & $2(50)$ \\
Peripheral neuropathy & $1(0,9)$ & $0(0)$ \\
Epilepsy & $5(4,5)$ & $2(40)$ \\
Spinal stenosis & $1(0,9)$ & $1(100)$ \\
Neuralgia & $2(1,8)$ & $1(50)$ \\
Motor neuron disease & $1(0,9)$ & $1(100)$ \\
Pseudotumor cerebri & $1(0,9)$ & $0(0)$ \\
Obstructive sleep apnea syndrome & $1(0,9)$ & $1(100)$ \\
Myopathy & $1(0,9)$ & $0(0)$ \\
RLS & $1(0,9)$ & $0(0)$ \\
\hline
\end{tabular}

*Percentage of all patients

*** Percentage of patients with neurological comorbidities (row)

Reasons for consultation are listed in Table 4. Altered mental status was the most common cause of consultation $(55 \%)$.

Thirteen patients with consultation had worsened encephalopathy due to a neurological comorbidity. Four had intracranial mass lesions, 2 had normal-pressure hydrocephalus, and 11 had dementia.

Twenty-six patients had new-onset encephalopathy. Three had hypoxic-ischemic lesions on MRI, and one had cortical laminar necrosis. One patient demonstrated central pontine myelinolysis on MRI. One patient had aseptic meningitis with normal findings and abnormal MRI suggesting meningeal involvement (Fig. 2). One patient had temporary global amnesia lasting for $3 \mathrm{~h}$ with normal MRI features.

One patient demonstrated encephalopathy on the 5th day of disease onset. She had action myoclonus, generalized rigidity and catatonia. CSF analysis showed 30 mononuclear lymphocytes with normal protein levels. Cranial MRI was unremarkable. EEG demonstrated bioelectrical generalized slow-wave activity. She was diagnosed with postinfectious encephalitis, and she was treated with intravenous methylprednisolone for 7 days and oral lorazepam $5 \mathrm{mg} /$ day. Complete recovery was achieved 2 weeks after.

Seven patients had previous parkinsonism, and their symptoms were exacerbated after COVID-19 infection; 3 of them died. One patient developed bilateral mild parkinsonian symptoms after mirtazapine treatment for insomnia. One had new-onset oromandibular dystonia.

Four patients with epilepsy had increased seizure frequency. One of them had central nervous system lymphoma, and one had intracranial metastases. One had an intracranial mass lesion and new-onset sinus vein thrombosis. One patient had inoperable glioblastoma multiforme and was diagnosed with status epilepticus. All of them died from causes other than epilepsy.

Five patients had new-onset seizures. One patient was diagnosed with ischemic stroke with multiple bilateral cerebral infarcts on MRI and developed status epilepticus (Fig. 3). Routine CSF analysis was unremarkable. The viral encephalitis panel and COVID-19 PCR in CSF were negative. She died in the intensive care unit due to septic shock. Another patient with uremic encephalopathy and new-onset seizures also died. One patient with a history of stroke had a seizure. Although she did not receive any antiepileptic treatment, she did not have a second seizure. Seizure etiology was unknown in the remaining two patients, as their evaluation did not reveal any central nervous system pathology. One patient who was under propofol infusion had a first-time seizure with cessation of infusion. She did not have a second seizure after restarting the infusion. Other patients did not experience a second seizure after levetiracetam treatment. Both patients died, not because of the seizures.

Four patients had a previous diagnosis of MG, one of whom required intravenous immunoglobulin treatment due
Table 3 Baseline characteristics of patient groups

\begin{tabular}{llll}
\hline & $\begin{array}{l}\text { Exacerbation of previous neuro- } \\
\text { logical disorder } n=45\end{array}$ & New-onset $n=67$ & $p$ \\
\hline Age median (IQR) & $68(54.5-76)$ & $69(58-77)$ & 0.482 \\
Gender (male) $n(\%)$ & $29(64.4)$ & $39(\% 58.2)$ & 0.508 \\
Hypertension $n(\%)$ & $12(26.7)$ & $24(35.8)$ & 0.309 \\
Diabetes mellitus $n(\%)$ & $9(20)$ & $12(17.9)$ & 0.781 \\
Malignancy $n(\%)$ & $7(15.6)$ & $10(14.9)$ & 0.927 \\
Cardiac disorders $n(\%)$ & $10(22.2)$ & $13(19.4)$ & 0.717 \\
Pulmonary disorder $n(\%)$ & $4(8.9)$ & $7(10.4)$ & 0.786 \\
Chronic renal failure $n(\%)$ & $5(11.1)$ & $6(9)$ & 0.707 \\
COVID severity $(>2) n(\%)$ & $24(53.3)$ & $47(70.1)$ & 0.07 \\
Exitus $n(\%)$ & $12(26.7)$ & $23(34.3)$ & 0.391 \\
\hline
\end{tabular}


Table 4 Reasons for neurological consultation*

\begin{tabular}{lllll}
\hline Reason for neurology consultation & $(n=112)$ & Exacerbation & New-onset & Exitus \\
\hline Altered mental status & 39 & 13 & 26 & 11 \\
Stroke & 21 & & 21 & 13 \\
Extrapyramidal symptoms & 9 & 7 & 2 & 3 \\
Headache & 10 & 4 & 6 & 1 \\
Seizure & 9 & 4 & 5 & 6 \\
Vertigo/dizziness & 6 & & 6 & 1 \\
Sensory disturbances & 5 & & 5 & 0 \\
Cranial neuropathy & 1 & 1 & 0 \\
Retinitis & 1 & & $2 * *$ & 0 \\
Myasthenia gravis & 6 & 4 & 3 & 2 \\
Hiccups & 3 & & 4 & 1 \\
Syncope & 4 & 1 & & 0 \\
Restless legs syndrome & 1 & 2 & & 0 \\
Neuralgia*** & 2 & 1 & & 1 \\
Motor neuron disease & 1 & & & 1 \\
\hline
\end{tabular}

*Patients consulted with more than one reason were counted multiple times

** Definitive diagnosis not established

**** One had trigeminal, one had occipital neuralgia
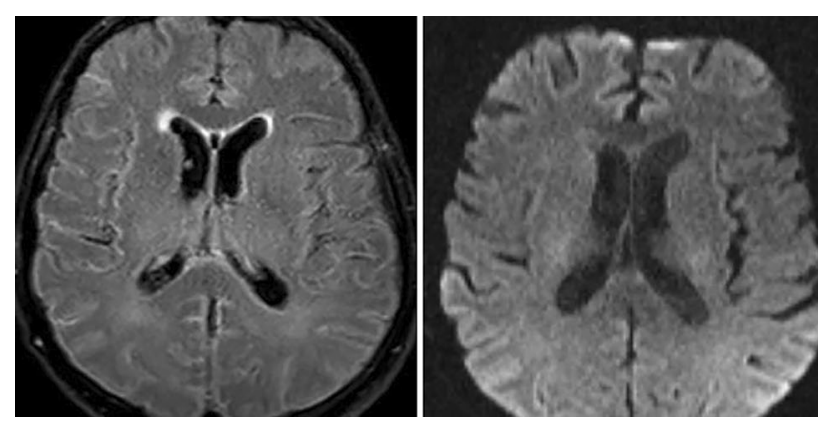

Fig. 2 Axial T2 flair image shows increased signal intensity in subarachnoid spaces and diffusion-weighted trace image is in normal limits to worsening of the bulbar symptoms. Two patients developed new-onset fluctuating diplopia, suggesting a neuromuscular junction disorder. One of them was hospitalized during the first wave, and her symptoms emerged after quinolone. However, none of them had a response to oral pyridostigmine. Unfortunately, a definitive diagnosis could not be achieved for these two patients after discharge.

Twenty-one patients had a stroke after COVID-19 infection (Fig. 4). One patient had a hemorrhagic stroke. One patient had sinus venous thrombosis (also mentioned above for seizures). The remaining 19 patients had an ischemic stroke. Two of them developed hemorrhagic transformation (Fig. 3). Two of the ischemic patients who were in the first wave did not receive anticoagulation before stroke onset. The rest of the patients were on low molecular weight heparin treatment when they had a stroke.
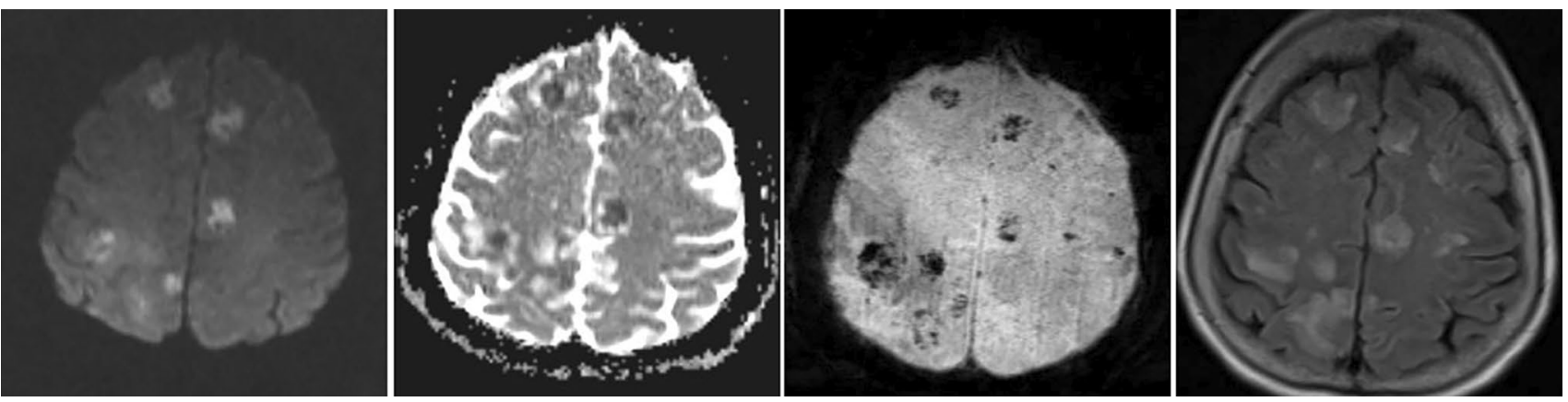

Fig. 3 Axial diffusion-weighted images including trace and ADC show multiple areas of restricted diffusion. Axial flair and SWI images are showing edematous and hemorrhagic nature of the lesions 

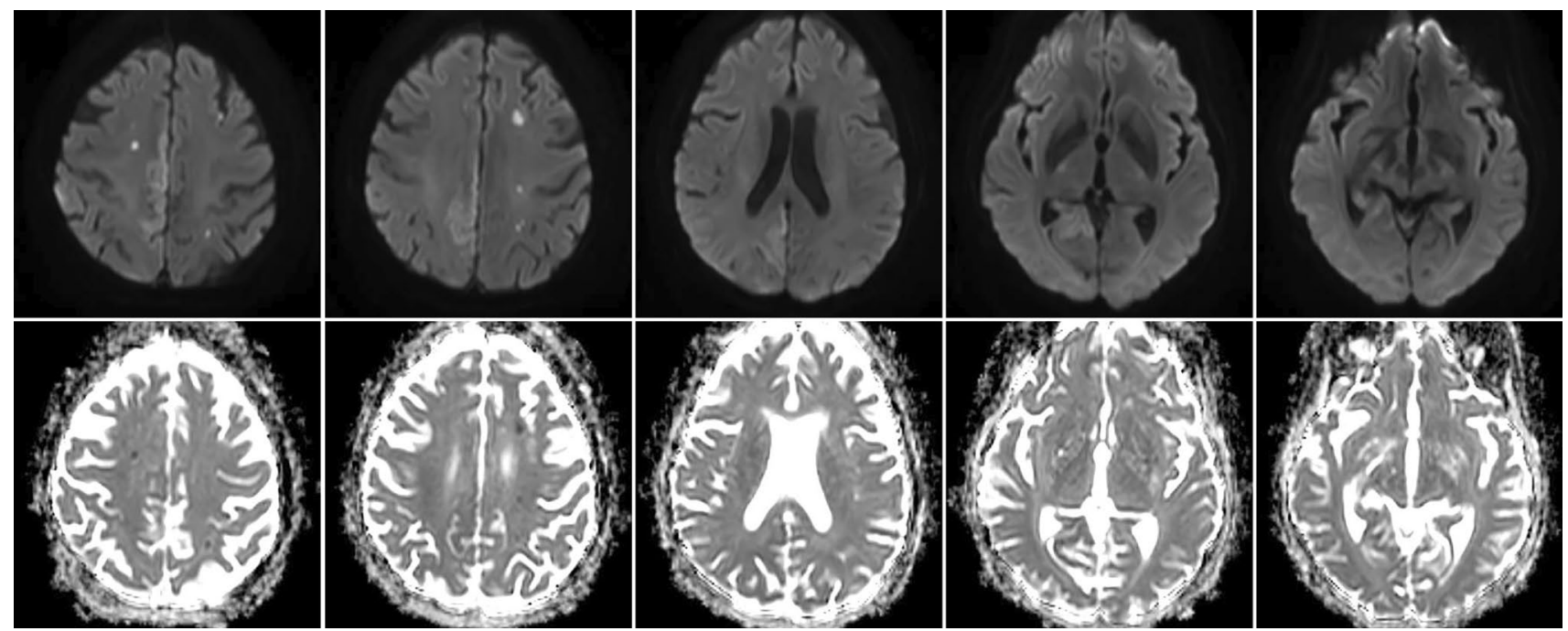

Fig. 4 Axial diffusion-weighted images including trace and ADC show multiple subcortical restricted diffusion abnormalities and right parafalcian cortico-subcortical lesion extending to ipsilateral hippocampus

Table 5 Laboratory findings of patients

\begin{tabular}{llll}
\hline & Exacerbation & New-onset & $p$ \\
\hline Ferritin $(\mathrm{ng} / \mathrm{mL})$ & $885.9(354.5-2000)$ & $1015(461.9-2000)$ & 0.63 \\
D-Dimer $(\mu \mathrm{g} / \mathrm{ml})$ & $3.25(1.39-5.5)$ & $4.63(1.46-12.8)$ & 0.2 \\
Fibrinogen $(\mathrm{mg} / \mathrm{dL})$ & $691(578.2-848.2)$ & $643.9(499.8-828)$ & 0.45 \\
Creatinine kinase & $155(79.2-322.2)$ & $268.5(106.7-$ & 0.02 \\
$\quad(\mathrm{U} / \mathrm{L})$ & & $999.2)$ & \\
Platelet $\left(10^{\wedge} 3 / \mu \mathrm{L}\right)$ & $201(140.6-251.6)$ & $216(151.2-282)$ & 0.58 \\
\hline
\end{tabular}

Three patients had intractable hiccups. Neurological evaluation of these patients was unremarkable. All of them had severe pneumonia in thorax CT scans, which may be the cause of hiccups by phrenic nerve irritation.

Two patients had new-onset blurred vision. One had macular edema in fundus examination. Routine CSF analysis was inconclusive. She was diagnosed with retinitis. One patient had abducens nerve palsy, but the etiology was obscure because the patient was not admitted to neurology after discharge.

Table 5 shows laboratory findings that may be related to neurological manifestations. Creatinine Kinase was higher in the new-onset patient group $(p<0.05)$.

\section{Discussion}

The major findings of this study are (i) severe neurological involvement of COVID-19 disease is uncommon in hospitalized patients, (ii) worsening of an ongoing neurological disorder or having a new-onset neurological disorder during the COVID-19 disease may cause higher mortality compared to nonneurological patients, (iii) stroke and dementia were the leading neurological diseases in hospitalized patients, and (iv) patients with a new-onset neurological disorder during the COVID-19 disease are more likely to have a more severe COVID-19 disease course and increased CK levels, which may reflect the severity of the disease.

In our cohort, 2257 patients were hospitalized during the study period, and only 127 (5.6\%) were consulted by a neurologist. Nonspecific mild neurological symptoms were not addressed within this study, as they were not serious enough to alert the clinician for consultation, especially in a pandemic situation. Consultations targeted obvious neurological deficits that occurred or worsened during the disease course. For this reason, this study proposes that serious neurological complications are rare in our experience. The incidence of neurological symptoms in COVID-19 disease varies between 1.6 and $36 \%$ depending on how neurological symptoms were evaluated. Studies with a similar design as ours reported similar rates, whereas studies including mild neurological symptoms reported up to 36\% [21-26].

To our knowledge, this is the first study investigating outcomes of neurological disease exacerbation and new-onset neurological conditions related to COVID-19 disease in hospitalized patients. Herman et al. aimed to analyze literature from the same point of view in which they searched the literature for COVID-19 patients with preexisting neurological conditions or patient-reported neurological complications during COVID-19 infection. They conducted a primary literature search and found that 322 of 4014 (8.0\%) patients had a preexisting neurological disease, and these patient groups, especially those with cerebrovascular event history, had an increased requirement for intensive care unit treatment and developed acute respiratory distress syndrome. They also 
mentioned various case reports of neurological involvement of COVID-19 infection and four retrospective studies demonstrating increased neurological complications of COVID19 infection, which are also discussed above. Unfortunately, they could not compare these two groups due to inconsistent reporting and limited statistical analysis [27]. In our cohort, age, sex, vascular risk factors and mortality rates were similar in these groups. COVID-19 disease was more severe in the new-onset neurological disorder patients $(p=0.07)$. On the other hand, severe COVID-19 disease increases the likelihood of having a new-onset neurological disorder. This finding was similar to a study in which patients with severe COVID-19 infection developed neurological involvement, such as cerebrovascular disease, altered mental status and skeletal muscle injury [25]. Therefore, patients with severe COVID-19 infection must be monitored closely for neurological manifestations.

Our results suggest that consultation by neurology, independent of exacerbation or new-onset neurological disorder, is related to high mortality in COVID-19. Mortality rates were $9.9 \%$ and $19.9 \%$ during the first and second waves in hospitalized COVID-19 patients, respectively. This twofold increase can be attributed to different indications for hospitalization in our hospital between the first and second waves; aiming to adjust health-care capacity to exponentially increased COVID-19 cases, the same measures were valid all over the world [28]. It is interesting that one-third of patients consulted by neurology died in both waves. Although this can be interpreted as having a severe neurological symptom that may be the reason for hospitalization, it may also indicate that patients with severe neurological symptoms face an increased risk of mortality irrespective of the burden of COVID-19 disease severity, considering that patients with relatively milder COVID-19 disease were hospitalized in the first wave. A cohort of 576 COVID-19 patients evaluated the impact of chronic neurological disorders on the prognosis of COVID-19 disease and their findings support this hypothesis as the presence of chronic neurological disorders was found to be an independent predictor of mortality in hospitalized COVID-19 patients apart from disease severity, although they did not evaluate new-onset neurological disorders [29].

Epilepsy patients constituted a small proportion in both groups, and the majority of them had cerebral involvement. Increased seizure frequency and new-onset seizures during COVID-19 have been reported previously, and our data were similar to these $[30,31]$.

Altered mental status and encephalopathy in COVID-19 patients have a broad clinical spectrum and various etiologies. Severe COVID-19 patients especially tend to develop altered mental status and encephalopathy but it is hard to differentiate whether it is a critical illness related encephalopathy, cytokine storm or specific to COVID-19 [32]. On the other hand, older patients with COVID-19 who are presented with delirium are also reported in the literature [33]. It was the most common reason for consultation and had a high rate of mortality in our study, as previous studies reported $[15,25]$.

An interesting finding of our study was consultations of intractable hiccups for 3 different patients, none of whom had abnormalities in their cranial imaging; instead, they had severe pneumonia, and one died. There are similar COVID19 cases presenting with intractable hiccups in the literature; some of these cases have accompanying symptoms such as sore throat and fever, but there are atypical cases presenting with only hiccups, and one persisted for 7 days. These reports focus on pneumonia rather than cranial pathology, such as involvement of the area postrema; even so, cranial imaging was performed in our cases, and none of them had involvement. Metoclopramide is partially beneficial, but actual improvement was achieved after down-trending inflammatory markers. As a result, it is wise to remember hiccups as a condition that should be considered in association with COVID-19 [34-36].

The major limitations of our study were (i) including only consulted COVID-19 patients, (ii) lack of information about mild neurological involvement, and (iii) inability to evaluate the outcomes of the patients. These were partly due to the retrospective nature of the study and partly because of pandemic conditions. Even though it satisfies our curiosity about the neurological involvement of COVID-19 patients in our experience, additional studies are necessary on this topic.

Funding None.

Availability of data and material The datasets analysed during the current study are available from the corresponding author on reasonable request.

\section{Declarations}

Conflict of interest MHS, UT, ÇÖ, SS, CA, ZEKG, GN, EK, MAS, $\mathrm{HA}, \mathrm{SB}, \mathrm{RG}$ and FKS declare that they have no conflict of interest.

\section{References}

1. Zhu N, Zhang D, Wang W et al (2020) A novel coronavirus from patients with pneumonia in China, 2019. N Engl J Med 382:727733. https://doi.org/10.1056/NEJMoa2001017

2. WHO Director-General's opening remarks at the media briefing on COVID-19 - 25 March 2020. https://www.who.int/directorgeneral/speeches/detail/who-director-general-s-opening-remarksat-the-media-briefing-on-covid-19---25-march-2020. Accessed 17 Apr 2021

3. Genel Koronavirüs Tablosu. https://covid19.saglik.gov.tr/TR66935/genel-koronavirus-tablosu.html. Accessed 17 Apr 2021 
4. Jin Y, Yang H, Ji W et al (2020) Virology, epidemiology, pathogenesis, and control of COVID-19. Viruses 12:372. https://doi. org/10.3390/v12040372

5. Rhea EM, Logsdon AF, Hansen KM et al (2021) The S1 protein of SARS-CoV-2 crosses the blood-brain barrier in mice. Nat Neurosci 24:368-378. https://doi.org/10.1038/s41593-020-00771-8

6. Gu J, Gong E, Zhang B et al (2005) Multiple organ infection and the pathogenesis of SARS. J Exp Med 202:415-424. https://doi. org/10.1084/jem.20050828

7. Zubair AS, McAlpine LS, Gardin T et al (2020) Neuropathogenesis and Neurologic Manifestations of the Coronaviruses in the Age of Coronavirus Disease 2019: A Review. JAMA Neurol 77:1018-1027. https://doi.org/10.1001/jamaneurol.2020.2065

8. Bolay H, Gül A, Baykan B (2020) COVID-19 is a Real Headache! Headache 60:1415-1421. https://doi.org/10.1111/head.13856

9. Saniasiaya J, Kulasegarah J (2021) Dizziness and COVID-19. Ear Nose Throat J 100:29-30. https://doi.org/10.1177/0145561320 959573

10. Avula A, Nalleballe K, Narula $\mathrm{N}$ et al (2020) COVID-19 presenting as stroke. Brain Behav Immun 87:115-119. https://doi.org/10. 1016/j.bbi.2020.04.077

11. Bagnato S, Boccagni C, Marino G et al (2020) Critical illness myopathy after COVID-19. Int J Infect Dis 99:276-278. https:// doi.org/10.1016/j.ijid.2020.07.072

12. Iaconetta G, De Luca P, Scarpa A et al (2020) Meningoencephalitis Associated with SARS-Coronavirus-2. Transl Med UniSa 23:42-47

13. Toscano G, Palmerini F, Ravaglia S et al (2020) GuillainBarré Syndrome Associated with SARS-CoV-2. N Engl J Med 382:2574-2576. https://doi.org/10.1056/NEJMc2009191

14. Sousa GJB, Garces TS, Cestari VRF et al (2020) Mortality and survival of COVID-19. Epidemiol Infect. https://doi.org/10.1017/ S0950268820001405

15. Bianchetti A, Rozzini R, Guerini F et al (2020) Clinical presentation of COVID19 in dementia patients. J Nutr Health Aging 24:560-562. https://doi.org/10.1007/s12603-020-1389-1

16. Cilia R, Bonvegna S, Straccia G et al (2020) Effects of COVID-19 on parkinson's disease clinical features: a community-based casecontrol study. Mov Disord 35:1287-1292. https://doi.org/10.1002/ mds. 28170

17. Fasano A, Antonini A, Katzenschlager R et al (2020) Management of advanced therapies in parkinson's disease patients in times of humanitarian crisis: The COVID-19 experience. Mov Disord Clin Pract 7:361-372. https://doi.org/10.1002/mdc3.12965

18. Guidon AC, Amato AA (2020) COVID-19 and neuromuscular disorders. Neurology 94:959-969. https://doi.org/10.1212/WNL. 0000000000009566

19. Parrotta E, Kister I, Charvet L et al (2020) COVID-19 outcomes in MS: Observational study of early experience from NYU Multiple Sclerosis Comprehensive Care Center. Neurology - Neuroimmunology Neuroinflammation. https://doi.org/10.1212/NXI.00000 00000000835

20. Organization WH (2020) Clinical management of COVID-19: interim guidance, 27 May 2020. World Health Organization

21. Cagnazzo F, Arquizan C, Derraz I et al (2021) Neurological manifestations of patients infected with the SARS-CoV-2: a systematic review of the literature. J Neurol 268:2656-2665. https://doi.org/ 10.1007/s00415-020-10285-9

22. Guan W, Ni Z, Hu Y et al (2020) Clinical characteristics of coronavirus disease 2019 in China. N Engl J Med 382:1708-1720. https://doi.org/10.1056/NEJMoa2002032
23. Mao L, Jin H, Wang M et al (2020) Neurologic manifestations of hospitalized patients with coronavirus disease 2019 in Wuhan, China. JAMA Neurol 77:683-690. https://doi.org/10.1001/jaman eurol.2020.1127

24. Sharifian-Dorche M, Huot P, Osherov M et al (2020) Neurological complications of coronavirus infection; a comparative review and lessons learned during the COVID-19 pandemic. J Neurol Sci 417:117085. https://doi.org/10.1016/j.jns.2020.117085

25. Yuksel H, Gursoy GT, Dirik EB et al (2021) Neurological manifestations of COVID-19 in confirmed and probable cases: A descriptive study from a large tertiary care center. J Clin Neurosci 86:97-102. https://doi.org/10.1016/j.jocn.2021.01.002

26. Amanat M, Rezaei N, Roozbeh M et al (2021) Neurological manifestations as the predictors of severity and mortality in hospitalized individuals with COVID-19: a multicenter prospective clinical study. BMC Neurol 21:116. https://doi.org/10.1186/ s12883-021-02152-5

27. Herman C, Mayer K, Sarwal A (2020) Scoping review of prevalence of neurologic comorbidities in patients hospitalized for COVID-19. Neurology 95:77-84. https://doi.org/10.1212/WNL. 0000000000009673

28. Xu S, Li Y (2020) Beware of the second wave of COVID-19. Lancet 395:1321-1322. https://doi.org/10.1016/S0140-6736(20) 30845-X

29. García-Azorín D, Martínez-Pías E, Trigo J et al (2020) Neurological comorbidity is a predictor of death in Covid-19 disease: A Cohort Study on 576 patients. Front Neurol 11:781-781. https:// doi.org/10.3389/fneur.2020.00781

30. Danoun OA, Zillgitt A, Hill C et al (2021) Outcomes of seizures, status epilepticus, and EEG findings in critically ill patient with COVID-19. Epilepsy Behav 118:107923. https://doi.org/10. 1016/j.yebeh.2021.107923

31. Zeng C, Meng H, Zhu Y et al (2021) Correlation of seizure increase and COVID-19 outbreak in adult patients with epilepsy: findings and suggestions from a nationwide multi-centre survey in China. Seizure 88:102-108. https://doi.org/10.1016/j.seizure. 2021.03.029

32. Helms J, Kremer S, Merdji H et al (2020) Neurologic features in severe SARS-CoV-2 infection. N Engl J Med 382:2268-2270. https://doi.org/10.1056/NEJMc2008597

33. Cipriani G, Danti S, Nuti A et al (2020) A complication of coronavirus disease 2019: delirium. Acta Neurol Belg 120:927-932. https://doi.org/10.1007/s13760-020-01401-7

34. Atiyat R, Veeraballi S, Al-Atiyat $\mathrm{N}$ et al (2021) A rare case report of persistent hiccups as an atypical presentation of COVID-19. Cureus 13:e13625. https://doi.org/10.7759/cureus.13625

35. Ikitimur H, Borku Uysal B, Ikitimur B et al (2021) Case report: two cases of persistent hiccups complicating COVID-19. Am J Trop Med Hyg. https://doi.org/10.4269/ajtmh.21-0190

36. Totomoch-Serra A, Ibarra-Miramon CB, Manterola C (2021) Persistent hiccups as main COVID-19 symptom. Am J Med Sci. https://doi.org/10.1016/j.amjms.2021.01.001

Publisher's Note Springer Nature remains neutral with regard to jurisdictional claims in published maps and institutional affiliations. 